\title{
Organização de um Serviço de Assistência ao Paciente com Diabetes Melito Tipo 1
}

\begin{abstract}
RESUMO
Neste artigo são apresentadas as bases para a organização de um serviço especializado em diabetes na infância e na adolescência. É mostrado o modo como foi implementado o Instituto da Criança com Diabetes do Rio Grande do Sul (ICD) e a esquematização de seu trabalho. Seus objetivos são: diminuir hospitalizações por situações agudas, diminuir a freqüência de complicações crônicas e capacitar recursos humanos. São atendidos, em regime ambulatorial e de hospital-dia, 1.315 pacientes, majoritariamente diabetes melito tipo 1, de maneira gratuita, pelo Sistema Único de Saúde (SUS), por meio de convênio com o Grupo Hospitalar Conceição, Ministério da Saúde. Um programa educativo diário é realizado, com aulas de 45 minutos de duração, que fazem parte da atenção ao paciente e à sua família. Mostra-se uma diminuição das internações hospitalares, de 2004 a 2007 , de $7,5 \%$ para 2,7\%. Também são apresentados os diversos esquemas terapêuticos seguidos pela equipe interdisciplinar do instituto no atendimento às diferentes faixas etárias. (Arq Bras Endocrinol Metab 2008;52/2:219-232)
\end{abstract}

Descritores: Diabetes melito tipo 1; Assistência; Equipe; Multidisciplinar; Educação

\begin{abstract}
Establishment of a Collaborative Work Team Management for Type 1 Diabetes Mellitus Patient.

This article will provide the necessary information to establish a childhood and adolescence management center that would promote the integration and coordination of interdisciplinary members as healthcare teams. It will also show how Instituto da Criança com Diabetes from Rio Grande do Sul (ICD) was built, structured and how it works. The aim of this program is to decrease the frequency of hospitalization in acute cases, to decrease chronic complications and to qualify human resources. So far 1315 outpatients and day-care hospital patients, mostly type 1 diabetes, have been seeing free of chargein Public Health Service (PHS), in a partnership with Grupo Hospitalar Conceição $(\mathrm{GHC})$ and the Ministry of Health. Among other activities an educational program (consisting of 45-minute daily classes) is given to the patient and his family. From 2004 to 2007 it could be seen a decrease from 7,5\% to $2,7 \%$ in the diabetes related hospitalization concerning these patients. Clinical guidelines followed by the interdisciplinary ICD team will also be presented in this essay. (Arq Bras Endocrinal Metab 2008; 52/2:219-232)
\end{abstract}

Keywords: Type 1 diabetes; Management; Team; Multidisciplinary; Education atualização

\author{
BALDUINo TsCHIEDEL \\ GisLaine VIsSOKY Cé \\ César Geremia \\ Paula MondadorI \\ Silvana Speggiorin \\ Marcia K. C. Puñales
}

Instituto da Criança com

Diabetes do Hospital da Criança Conceição do Grupo Hospitalar Conceição do Ministério da Saúde, Porto Alegre, RS, Brasil.
Recebido em 10/12/2007 Aceito em 20/12/2007 


\section{INTRODUÇÃO}

O DIABETES MELITO TIPO 1 (DMl), embora com incidência muito inferior ao diabetes melito tipo 2 (DM2), responde por 65 mil novos casos a cada ano, mundialmente (1). O estudo The Diabetes Control and Complications Trial Research Group (2) mostrou que o tratamento intensivo do DMl inquestionavelmente leva à redução das complicações microvasculares do diabetes, mas que esta intensificação do tratamento necessita efetivo automanejo, que requer processo educativo freqüente, com adequado suporte continuado (3-5). Embora algumas revisões demonstrem que intervenções educacionais tenham pequeno a médio efeito benéfico no controle glicêmico, elas parecem ter efeito maior nos desfechos psicológicos (6-8). Entretanto, aqueles pacientes que não recebem educação específica são mais propensos a desenvolver complicações relacionadas ao diabetes $(3,9,10)$.

De acordo com a International Society for Paediatric and Adolescent Diabetes (ISPAD), os centros de diabetologia pediátrica devem assegurar cuidados médicos especializados, preferencialmente em regime ambulatorial, e a educação é a chave para o manejo bem-sucedido do diabetes. Um processo educativo estruturado, com equipe interdisciplinar treinada, deveria estar disponível a todos os pacientes com diabetes no momento do diagnóstico, e então ser oferecido de modo continuado $(3,11,12)$.

Neste artigo, será apresentado o trabalho que é desenvolvido pelo Instituto da Criança com Diabetes do Rio Grande do Sul (ICD), uma organização não-governamental que atende pacientes com DM na infância e na adolescência, principalmente DMI $(95,9 \%)$, pelo Sistema Único de Saúde (SUS), por meio de convênio com o Grupo Hospitalar Conceição (GHC), complexo hospitalar de Porto Alegre pertencente ao Ministério da Saúde. Os objetivos do ICD são: diminuir hospitalizações por situações agudas (hipoglicemias e cetoacidoses), diminuir a freqüência de complicações crônicas e capacitar recursos humanos. Essa parceria com o GHC permite que os pacientes sejam atendidos de maneira gratuita pelo SUS, com facilidades na marcação de consultas e acesso à equipe interdisciplinar, contratada por concurso público, até mesmo com acesso pelo telefone. Permite, também, que muitos desses pacientes sejam alcançados pelos insumos e pelas técnicas diagnósticas e terapêuticas não previstos pelo SUS, conseguidos por recursos do ICD, oriundos de captação na comunidade. Além disso, permeia-se o atendimento interdisciplinar com um processo educativo diário, com aulas integradas durante o atendimento. Essas aulas foram elaboradas pela equipe técnica do ICD, mas sofreram o crivo de uma equipe de educadoras do curso de pós-graduação em Educação da Pontifícia Universidade Católica do Rio Grande do Sul. Embora as diferentes formas de captação de recursos não sejam o escopo deste artigo, pode-se ressaltar que o carro-chefe é a "Corrida para Vencer o Diabetes", evento comunitário já incorporado ao cronograma da Prefeitura Municipal de Porto Alegre, e que no ano de 2007, em sua 9a edição, vendeu 33 mil camisetas.

A incidência de DMl vem aumentando mundialmente, afetando crianças em idades cada vez mais precoces. No Estado do Rio Grande do Sul, estima-se que existam 8 mil crianças e adolescentes com DMl e 312 novos casos sejam diagnosticados anualmente. O ICD foi inaugurado em 19 de janeiro de 2007, e atualmente acolhe 1.315 crianças e adolescentes com idades entre 0 e 20 anos ao diagnóstico de DM, tendo realizado o atendimento de 124 novos casos $(39,7 \%$ da estimativa do RS) de DMl no último ano.

As primeiras consultas são agendadas por telefone ou pessoalmente, e o primeiro atendimento é realizado no hospital-dia, independentemente do tempo de diagnóstico do diabetes. Ao chegarem pela primeira vez na instituição, todos os pacientes são avaliados inicialmente pelo endocrinologista, porquanto crianças e adolescentes até 15 anos também são atendidos pelo pediatra. Nessa primeira consulta, são atendidos pela equipe de enfermagem, que fornecem orientações quanto aos locais de aplicação e preparo das insulinas, automonitorização e avaliação de lipodistrofias, pelos nutricionistas, que oferecem prescrição de planos alimentares balanceados e individualizados, privilegiando a contagem de carboidratos sempre que possível. Também são entrevistados pela assistente social, cujo atendimento sociofamiliar visa a avaliação, o apoio, a orientação e o acompanhamento, no que diz respeito aos insumos e à educação em saúde. Os pacientes são reagendados no hospitaldia para reavaliação, de acordo com necessidades individuais, para o dia ou para a semana seguinte. Nessa consulta, são realizados os ajustes terapêuticos, a verificação dos resultados das glicemias capilares, a aplicação da insulina e o esclarecimento de dúvidas, principalmente naqueles com diagnóstico mais recente. 
Posteriormente, os pacientes são encaminhados para o ambulatório, com atendimentos pela equipe interdisciplinar. Os dados dos pacientes (história médica, exame físico, achados laboratoriais, manejo terapêutico e seguimento) são inseridos por todos os profissionais em um software personalizado.

\section{ATENDIMENTO INTERDISCIPLINAR}

Os pacientes são atendidos por uma equipe interdisciplinar (médicos: endocrinologista, pediatra, oftalmologista, nefrologista, cirurgião e ginecologista; enfermeiro, nutricionista, psicólogo, assistente social, dentista e educador físico), têm direito a consultas mensais, bimestrais ou trimestrais e/ou atendimento e acompanhamento em hospital-dia, de acordo com os protocolos próprios de acompanhamento e o atendimento aos pacientes com diabetes, visando a atingir as metas de tratamento intensivo. Deve-se ressaltar que todos os pacientes com DMl são orientados ao tratamento intensivo, apenas não o fazendo no período de lua-de-mel.

\section{ATENDIMENTO AMBULATORIAL}

O atendimento ambulatorial é realizado por meio de consultas diárias nos turnos da manhã e da tarde, com a equipe interdisciplinar, com consultas mensais, bimestrais ou trimestrais, de acordo com as necessidades individuais. As consultas são agendadas para aproximadamente três profissionais. No entanto, os pacientes provenientes do interior do Estado, outros Estados ou aqueles com dificuldades específicas podem realizar seu atendimento com mais profissionais em um mesmo dia.

Dos 1.315 adolescentes e crianças com diabetes, $95,9 \%(1.261 / 1.315)$ apresentam DMl , 2,4\% (32/1.315) DM2, 0,9\% MODY (12/1.315) e 0,8\% (10/1.315) apresentam outras formas de diabetes melito. Os pacientes são procedentes de diferentes localidades $(25,7 \%$ de Porto Alegre, $32,5 \%$ da grande Porto Alegre, $40,8 \%$ do interior do Rio Grande do Sul, $0,9 \%$ de outros Estados e $0,1 \%$ de outros países), sendo 50,3\% (661/1.315) do sexo feminino. A média de idade atual dos pacientes é de $15,3 \pm 6,2$ anos, variando de 2,0 a 42,4 anos e ao diagnóstico foi de $9,1 \pm 5,1$ anos, variando de 1,5 meses a 29,4 anos. O tempo médio de duração do diabetes é de $6,2 \pm 5,1$ anos (mediana: 4,7 anos), variando de um diagnóstico recente a 34 anos de diabetes.
Em 2004, foram realizados no ambulatório, pela equipe interdisciplinar, 9.737 atendimentos interdisciplinares (total: 584 pacientes, com média de $811,4 \pm$ 250,0 atendimentos/mês, média de $243,5 \pm 57,0$ pacientes/mês e 12,2 $\pm 3,4$ pacientes/dia). Em 2005, foram realizados 15.568 atendimentos (total: $878 \mathrm{pa}$ cientes, com média de 1.297,3 $\pm 270,2$ atendimentos/ mês, média de $342,0 \pm 57,3$ pacientes/mês e $18,1 \pm$ 2,2 pacientes/dia). Em 2006, foram realizados 17.752 atendimentos (total: 1.116 pacientes, com média de $1.479,3 \pm 263,9$ atendimentos/mês, média de $359,1 \pm$ 55,2 pacientes/mês e $18,5 \pm 2,1$ pacientes/dia), e até 31 de outubro de 2007, foram realizados 15.342 atendimentos pela equipe interdisciplinar (total: 1.315 pacientes, com média de 1.534,2 $\pm 338,6$ atendimentos/ mês, média de $371,0 \pm 80,2$ pacientes/mês e $17,8 \pm$ 3,1 pacientes/dia).

\section{Endocrinologia}

$\mathrm{O}$ atendimento pelo endocrinologista é realizado diariamente nos turnos da manhã e da tarde, em regime ambulatorial ou hospital-dia (consultas ou internações/dia). $\mathrm{O}$ atendimento às primeiras consultas é realizado na unidade do hospital-dia. No ambulatório, as consultas são agendadas geralmente a cada três meses. No entanto, para pacientes com diagnóstico mais recente, ou em fase de lua-de-mel, as consultas são reagendadas com maior freqüência, podendo ser mensais. Nas consultas, são avaliados os resultados das glicemias capilares (automonitorização domiciliar) e realizados os ajustes nas doses de insulina, de acordo com esses valores. A automonitorização é recomendada três ou mais vezes ao dia: antes das principais refeições, duas horas após as refeições e antes de deitar (realizadas periodicamente), na madrugada (uma ou duas vezes por semana, às 3 horas ou às 4 horas) e em situações especiais (atividade física, dias de doença, aniversários, sintomas de hipo/hiperglicemia). Verificam-se os episódios de hipoglicemias e enfatizam-se os procedimentos diante desses episódios. São avaliados os resultados dos exames, principalmente de hemoglobina glicada Alc e se este se correlaciona com os valores obtidos na monitorização pela glicemia capilar. São checados os monitores e, sempre que possível, realiza-se o download dos resultados em um software específico, permitindo a discussão desses dados com os pacientes e seus familiares. 


\section{Pediatria}

$O$ pediatra realiza $o$ atendimento de crianças e adolescentes de 0 a 15 anos, diariamente, no turno da manhã no ICD. As consultas são realizadas conforme agendamento prévio ou atendimento de urgência. As reconsultas são agendadas a cada três meses, juntamente à equipe interdisciplinar, conforme necessidades individuais.

Todos os pacientes, na faixa etária anteriormente descrita, são encaminhados ao pediatra para monitorar o crescimento pôndero-estatural, orientar sobre puberdade e abordar a importante questão da anticoncepção efetiva a partir da adolescência, enfatizar o calendário oficial de vacinas e recomendar a imunização adicional para pneumococo, influenza (anualmente) e varicela. $\mathrm{O}$ pediatra também estimula o aleitamento materno, a fim de postergar a introdução de cereais e proteína animal para os primeiros seis meses de idade, recomenda hábitos de vida saudável, como a prática de atividade física regular e planejada, auxilia nas orientações escolares, diagnostica, solicita exames laboratoriais e de imagem e acompanha outras doenças, como epilepsia ou crise convulsiva, anemias, enteroparasitoses e patologias auto-imunes, como a doença celíaca ou a tireoidite de Hashimoto, e realiza o contato e o encaminhamento, quando necessários, para as diferentes especialidades pediátricas do Hospital da Criança Conceição. O pediatra executa um papel importante dentro da equipe interdisciplinar, pois em dias de doença (infecções) intensifica a importância da automonitorização e a boa hidratação, fica atento à preocupação excessiva com a auto-imagem e perda de peso das adolescentes, auxilia o paciente e sua família com os desafios de lidar com uma doença crônica que exige cuidados permanentes e impõe tantas restrições.

\section{Enfermagem}

As consultas com a enfermeira no ICD são realizadas individualmente em consulta ambulatorial ou em hospital-dia. São realizadas orientações gerais sobre técnica e locais de aplicação de insulina, materiais apropriados de preparo, conservação, transporte, validade, descarte do material pérfuro-cortante e biológico, além de orientação e conduta nos episódios de hipoglicemia. Essas consultas inicialmente são mais freqüentes e posteriormente se adaptam às outras consultas com a equipe interdisciplinar, a cada três meses.

No ICD, segue-se a técnica de conservação e armazenamento das insulinas, de acordo com as recomenda- ções do fabricante, mantendo os frascos ou cartuchos fechados sob refrigeração de $2{ }^{\circ} \mathrm{C} \mathrm{a} 8{ }^{\circ} \mathrm{C}$ e aqueles em uso entre $4{ }^{\circ} \mathrm{C}$ e $30^{\circ} \mathrm{C}$. Depois de aberto, o frasco ou cartucho, recomenda-se seu uso em um período máximo de 45 dias. A reutilização de seringas e agulhas, apesar de ser um assunto polêmico, é realizada na prática clínica, sendo importante orientar quanto ao descarte destas no caso de ausência de fio, tortuosidade ou contato com outras superfícies além da pele, assim como cuidados básicos de higiene.

O rodízio planejado dos locais de aplicação de insulina é importante para evitar as lipodistrofias, freqüentes em pacientes com DMl em tratamento intensivo. Sabe-se que para garantir a efetividade da insulinoterapia é recomendável discutir com o paciente a melhor opção de rodízio. No ICD, orienta-se realizar o rodízio dos locais de aplicação de insulina a cada três dias em lactentes e crianças até 7 anos de idade e a cada cinco a sete dias em adolescentes e adultos. Outra maneira utilizada para o rodízio é de acordo com as refeições (por exemplo, antes do café aplicar no braço, antes do almoço no abdome, antes do jantar na coxa e antes de deitar na região glútea). A técnica de automonitorização é demonstrada e ensinada seguindo os horários recomendados pelo médico. São orientados cuidados gerais de higiene e em especial com os pés, principalmente nos pacientes em puberdade ou com diagnóstico de diabetes há mais de cinco anos ou na presença de complicações crônicas associadas, visando a prevenção do pé diabético.

\section{Nutrição}

As orientações nutricionais no ICD são realizadas individualmente em consultas ambulatoriais de rotina ou no hospital-dia, ou em grupo, na oficina de nutrição. A maioria dos pacientes realiza consultas ambulatoriais a cada três meses, podendo ser semanais, quinzenais ou mensais, consoante as necessidades individuais.

$\mathrm{Na}$ primeira consulta, independentemente de orientação prévia, realiza-se a anamnese alimentar, a avaliação do perfil metabólico e dos dados antropométricos. Nesse momento, também é verificada a necessidade de uma orientação nutricional específica, caso existam complicações crônicas do DM ou intolerâncias alimentares. Inicialmente, recomenda-se a terapia nutricional convencional e, posteriormente, define-se qual o melhor método a ser seguido, podendo manter-se o convencional ou iniciar a contagem de carboidratos, de acordo com o perfil, o grau de conhecimento e enten- 
dimento, a disciplina e o desejo do paciente e/ou seus responsáveis. Elabora-se o plano alimentar individualizado, por escrito, e agenda-se um retorno em 15 a 30 dias, para reavaliar e verificar a adesão ao tratamento nutricional. Nesse momento, verificam-se as variações no peso corporal, os registros de glicemias capilares e os exames laboratoriais. Conforme os resultados, mantémse o plano vigente ou realizam-se modificações necessárias, bem como o reforço verbal e a motivação para o seguimento do plano prescrito.

Em situações de diagnóstico recente de $\mathrm{DMl}$, a terapia nutricional recomendada deve levar em consideração que esses pacientes podem se encontrar debilitados ou desnutridos pela perda de peso, podendo fazer parte do quadro clínico inicial de diagnóstico, sendo necessária a prescrição de plano alimentar que promova o reequilíbrio nutricional.

\section{Odontologia}

Na primeira consulta odontológica no ICD, é realizada a anamnese com perguntas específicas do estado geral do paciente, tipo de diabetes, data do diagnóstico, glicemia capilar, controle de placa e exame odontológico. As manifestações bucais associadas ao diabetes, como xerostomia, alterações de microflora bucal, atrofia de papilas, cáries, infecções, entre outras, são diagnosticadas e registradas na ficha clínica do paciente. No exame odontológico, são avaliadas as estruturas dentais, quanto às cáries ativa e inativa, restaurações e dos dentes ausentes ou extraídos. Quando necessários, são solicitados exames radiográficos, para avaliação de estruturas dentais e ósseas que possam auxiliar no diagnóstico e no plano terapêutico. A higiene bucal é avaliada pela presença de placa bacteriana (biofilme), com um controle supervisionado dela (escovação e fio dental). É realizada uma avaliação minuciosa quanto à presença de doença periodontal, cálculo salivar, sangramento gengival, mobilidade dental e bolsa periodontal. Os índices CPO-D (dentes permanentes cariados, perdidos, obturados) e/ ou CEO-D (dentes decíduos cariados, extraídos, obturados) são verificados conforme a idade do paciente.

Em consultas subseqüentes, antes de qualquer procedimento clínico, recomenda-se o controle supervisionado de biofilme bacteriano e bochechos com solução aquosa de clorexidina a $0,12 \%$. As revisões odontológicas ocorrem a cada trimestre. Em caso de procedimentos odontológicos prolongados, principalmente em horário de refeições ou na presença de sinais e sintomas de hipoglicemia, o trabalho é interrompido para realização de uma refeição leve ou para tratamento de hipoglicemia. Após a correção da glicemia, prossegue-se a consulta. Nos casos emergenciais, que apresentem processos infecciosos agudos, como abscessos dentários ou periodontais, é de grande importância o uso de antibióticos.

\section{Psicologia}

Os pacientes atendidos por psicólogos são encaminhados pela equipe interdisciplinar, por solicitação de seus responsáveis ou por procura espontânea do paciente. Os adolescentes e os adultos jovens buscam o atendimento por questões de seu cotidiano, conflitos da adolescência e de relacionamentos afetivos. Dificilmente o motivo da consulta é para tratar especificamente do diabetes.

$\mathrm{O}$ atendimento individual é realizado no ambulatório, com consultas agendadas, e/ou no hospital-dia como consulta extra. Na primeira consulta são atendidos os pacientes acompanhados de seus responsáveis. A partir desse primeiro contato, se estabelece a conduta de atendimento, podendo ser em consultas individualizadas ou com alguns encontros concomitantes com os pais e/ou responsáveis. As reconsultas são agendadas, com os demais profissionais da equipe interdisciplinar, em média a cada três meses. Em situações mais graves, o acompanhamento psicológico depende da procedência do paciente. Aqueles provenientes de Porto Alegre e grande Porto Alegre são agendados em consultas extras semanais ou quinzenais e aqueles do interior do Estado, apor causa da impossibilidade de comparecerem mais seguidamente às consultas, são encaminhados para o atendimento em suas cidades de origem, mas mantendo as reconsultas a cada três meses no ICD. Os pacientes que necessitam de avaliação e acompanhamento psiquiátrico são atendidos por profissionais do Grupo Hospitalar Conceição, nas instalações do ICD.

\section{Assistente social}

$\mathrm{O}$ atendimento e o acolhimento dos pacientes e seus cuidadores é realizado pela assistente social na primeira consulta ao ICD. Esse acolhimento é fundamental para o seguimento do tratamento, para o conhecimento do contexto paciente-família e adequação do tratamento a sua realidade. Nesse primeiro contato, é realizada a avaliação psicossocial e obtêm-se os dados referentes a moradia, renda familiar e escolaridade dos pacientes e de seus familiares. Em situações específicas, de baixa renda, são fornecidos alguns insumos até a família providenciar a documentação necessária para encaminhar a so- 
licitação destes à Secretaria Estadual ou Municipal da Saúde.

A assistência é abordada no sentido de informar e orientar quanto aos recursos da comunidade, acompanhamento dos processos relativos aos insumos, conforme a Portaria $n^{\circ}$ 074/2002 da Secretaria da Saúde do Estado do Rio Grande do Sul, criando uma rede de apoio de fundamental importância para o bom controle do DMl. Nesse seguimento faz-se o trabalho de busca ativa, ou seja, ir ao encontro de pacientes que estão sem comparecer aos atendimentos, com o intuito de saber os motivos das faltas e atuar nessa questão. Os contatos com as secretarias de saúde dos municípios do RS são realizados conforme a procedência dos pacientes, a fim de organizar o encaminhamento da documentação dos processos de insumos e/ou outras questões, dependendo de cada situação. Também é realizado o contato com escolas, conselho tutelar ou outra instituição que envolva nossos pacientes.

\section{Educador físico}

O objetivo do educador físico é incentivar, estimular, orientar, supervisionar e direcionar a prática do exercício físico nos pacientes com DM, com orientações teórico-práticas, individuais e em grupo, proporcionando melhor qualidade de vida. Essas consultas são realizadas de acordo com os encaminhamentos da equipe interdisciplinar ou por solicitação do paciente e de seus familiares. No primeiro atendimento, é realizada uma entrevista para obtenção de dados gerais, aferição das medidas antropométricas, pregas cutâneas, circunferência abdominal e índice de massa corporal. São realizadas orientações quanto à importância da prática de atividade física planejada e regular, alongamentos e esclarecimentos sobre a conduta diante da alimentação adequada durante a prática de atividades.

Outro objetivo do atendimento do educador físico é formar grupos de pacientes em atividades orientadas dentro do ICD, como Pilates, alongamento, ginástica geral, voleibol, basquetebol, futebol e caminhadas. Em longo prazo, o intuito é formar equipes para competições regionais, nacionais e internacionais.

\section{Oftalmologia}

A avaliação oftalmológica (exame de fundo de olho sob midríase medicamentosa com oftalmoscopia binocular indireta) é realizada ao diagnóstico em todos os pacientes e, posteriormente, repetida anualmente em pacientes com mais de três anos de diabetes ou na puberdade e, em casos específicos, com maior freqüência. Os pacientes com queixas oculares ou diminuição da acuidade visual podem marcar consultas conforme suas necessidades individuais.

Após atingida a midríase, a retina é avaliada por oftalmoscopia binocular indireta e por biomicroscopia do segmento posterior na lâmpada de fenda. Nos casos em que é detectada a retinopatia diabética, procede-se à documentação fotográfica por meio de retinografia colorida e com filtro verde em um sistema digital de imagens, o que facilita o posterior acompanhamento da evolução da doença. A angiografia fluoresceínica é realizada apenas em caso de dúvida quanto ao diagnóstico ou tratamento. A fotocoagulação a laser é indicada de acordo com os vários padrões e dependendo das alterações na retina, levando-se também em consideração a velocidade de progressão da retinopatia, a evolução da doença no olho contralateral e a possibilidade de manter um adequado acompanhamento do paciente.

O centro oftalmológico possui tecnologia adequada para tratamento da retinopatia diabética, realização de retinografia digitalizada e fotocoagulação a laser, os quais são realizados nas instalações do ICD. Outros exames complementares que possam ser necessários e as intervenções cirúrgicas são realizados no Grupo Hospitalar Conceição.

\section{Ginecologia e obstetrícia}

$\mathrm{O}$ atendimento pelo ginecologista é realizado uma vez por semana e as pacientes são agendadas conforme solicitação e necessidades individuais. São realizados atendimentos de rotina e orientações quanto à importância da anticoncepção e à prevenção de doenças sexualmente transmissíveis. Portanto, a gestação deve ser planeja$\mathrm{da}$, discutida com a equipe interdisciplinar, os riscos conhecidos e minimizados pela otimização do controle metabólico (Alc desejável < 6,5\%) e por cuidados de pré-concepção (fotocoagulação profilática da retina, reposição com folato etc.).

As gestantes são encaminhadas para o atendimento na unidade de alto risco do Grupo Hospitalar Conceição e realizam o perfil glicêmico no hospital-dia do ICD. Após o nascimento da criança, essas pacientes são reencaminhadas ao ICD.

\section{Nefrologia}

O rastreamento para nefropatia é realizado ao diagnóstico do diabetes, com o objetivo de excluir patologias 
renais prévias. Posteriormente, é realizado o rastreamento anual em pacientes com mais de cinco anos de diabetes ou na puberdade, com dosagens de microalbuminúria de amostra, pelo método de imunoturbidimetria (Cobas Integra $700^{\circledR}$, Roche), com valor de referência de até $29 \mathrm{mg} / \mathrm{L}$.

Em casos de microalbuminúria positiva na amostra, solicita-se microalbuminúria e creatinúria em urina de 24 horas (duas coletas com intervalo de três meses) e, se ambas forem acima de $20 \mu \mathrm{g} / \mathrm{min}$, inicia-se a terapêutica com droga inibidora da enzima conversora de angiotensina, além de orientação nutricional específica. Pacientes com macroalbuminúria e/ou perda de função renal são encaminhados para avaliação com nefrologista e também para avaliação com cirurgião especializado do serviço, visando a futuros transplantes.

$\mathrm{O}$ atendimento é realizado uma vez por semana nas instalações do ICD e os pacientes são acompanhados segundo as necessidades individuais, com retornos mensais, bimensais, trimestrais ou semestrais.

\section{ATENDIMENTO EM HOSPITAL-DIA}

Esta unidade possui seis leitos e três poltronas e realiza atendimento em forma de consultas, internação/dia ou atendimento telefônico (hotline), funcionando das 8 às 17 horas de segunda a sexta-feira. Os pacientes que apresentam controle metabólico inadequado, níveis de Alc $\geq 13 \%$, nas primeiras consultas, ou aqueles que não realizam automonitorização domiciliar são agendados e atendidos em regime de hospital-dia. São realizadas as consultas de urgências pediátricas e clínicas dos pacientes que estão em acompanhamento no ICD, como dias de doença (infecções: vias aéreas, urinária, dentária, cutânea, gastrintestinal) e outras causas.

Nesta unidade, os pacientes inicialmente são atendidos pela equipe de enfermagem com obtenção dos sinais vitais (FC, FR, TA e Tax), dados antropométricos (peso e altura) e medição da glicemia capilar. Posteriormente, são avaliados pelo endocrinologista de plantão na unidade, com ajustes do esquema terapêutico de acordo com os resultados da automonitorização domiciliar ou sintomatologia de hipo ou hipergicemia. A seguir, a enfermeira realiza as orientações quanto a aplicação de insulina, rodízio dos locais de aplicação, noções sobre os diferentes tipos de insulina e mistura desta, horários de aplicação e realização da monitorização pela glicemia capilar. Os pacientes também são ava- liados pela nutricionista, com prescrição da terapia nutricional e, em casos de necessidades específicas, ou primeiras consultas, são atendidos pela assistente social, que orienta quanto ao procedimento para a solicitação dos insumos. Durante o período de permanência no hospital-dia, são realizadas as monitorizações pré e pósprandiais (duas horas), a administração das insulinas conforme resultado das glicemias e/ou contagem de carboidrato e são oferecidas as refeições (plano alimentar balanceado) confeccionadas no serviço. Os pacientes também são acompanhados por pedagogas em atividades lúdicas e participam da brinquedoteca itinerante.

Nessa unidade é realizado o primeiro atendimento de cetoacidose diabética, com hidratação endovenosa rápida e insulina em bomba, controle de sinais vitais $\mathrm{e}$ diurese. A avaliação por profissionais de outras especialidades, como psiquiatria, urologia, cirurgia vascular e geral, gastroenterologia adulto e pediátrica é feita nessa unidade, caso necessária.

Em casos de necessidade de internação hospitalar plena (cetoacidose diabética, hipoglicemias graves, dias de doença ou outras causas), os pacientes são encaminhados aos serviços de UTI do Hospital da Criança Conceição (abaixo de 12 anos) ou Emergência do Hospital Conceição (acima de 12 anos).

Desde sua inauguração, o hospital-dia tem realizado atendimentos em forma de consultas e/ou internações/ dia. Em 2004, foram realizados 1.837 atendimentos (952 consultas e 885 internações/dia, cujas causas foram: $76,6 \%$ controle glicêmico, $11,3 \%$ hiperglicemia, $3,1 \%$ hipoglicemia, $5,2 \%$ diagnósticos recentes/primeiras consultas, $0,9 \%$ cetoacidose, $1,2 \%$ dias de doença e $1,7 \%$ por outras causas). Em 2005, foram realizados 1.962 atendimentos (625 consultas e 1.337 internações/ dia, causas: $83,9 \%$ controle glicêmico, $5,8 \%$ hiperglicemia, $1,95 \%$ hipoglicemia, 3,9\% diagnósticos recentes/primeiras consultas, $1,1 \%$ cetoacidose, $1,4 \%$ gestação, $0,6 \%$ dias de doença e 1,4\% por outras causas). Em 2006, foram realizados 1.850 atendimentos (918 consultas e 932 internações/dia, causas: 70,4\% controle glicêmico, 9,8\% hiperglicemia, 3,0\% hipoglicemia, 2,5\% cetoacidose, $2,4 \%$ gestação, $6,4 \%$ diagnósticos recentes/primeiras consultas, $3,6 \%$ dias de doença e $1,9 \%$ por outras causas). Até 31 de outubro de 2007 foram realizados 1.488 atendimentos (835 consultas e 653 internações/dia, causas: $61,5 \%$ controle glicêmico, $5,2 \%$ hiperglicemia, $1,7 \%$ hipoglicemia, $4,2 \%$ cetoacidose, $0,9 \%$ gestação, $21,9 \%$ diag- 
nósticos recentes/primeiras consultas, 2,6\% dias de doença e $2,0 \%$ por outras causas).

No ano de 2004 estavam em atendimento 587 pacientes, sendo 7,5\% $(44 / 587)$ desses pacientes encaminhados para internações hospitalares plenas. Em 2005, 2006 e até 31 de outubro de 2007, 4,1\% (36/878), $3,0 \%(34 / 1116)$ e $2,7 \%(35 / 1315)$ foram encaminhados para internação, respectivamente $(\mathrm{p}<0,001)$ ( $\mathrm{Fi}^{-}$ gura 1). Esses dados evidenciam a diminuição das hospitalizações dos pacientes em acompanhamento no ICD, objetivo principal da instituição.

\section{Atendimento a ligações telefônicas (hotline)}

$\mathrm{O}$ atendimento às ligações telefônicas é realizado diariamente das 8 às 17 horas, pelo médico endocrinologista e/ou pela enfermeira responsável pelo hospital-dia e das 8 às 12 horas e 30 minutos também são realizados pela médica pediatra ou pela assistente social (orientações gerais quanto a obtenção de insumos, encaminhamento de laudos e andamento dos processos), de acordo com as necessidades específicas. $\mathrm{O}$ cadastro dessas ligações começou a partir do dia 2 de janeiro de 2006 e, até o dia 31 de outubro de 2007 , foram recebidas 2.320 ligações ( 1.183 no ano de 2006 e 1.137 até 31 de outubro de 2007 ), com média de $105,4 \pm 27,8$ ligações por mês. Os motivos das ligações telefônicas foram: $50,6 \%$ por orien- tações gerais (dúvidas quanto aos insumos, encaminhamento de laudos, orientação quanto à aplicação de insulina, validade, canetas aplicadoras, troca de cartuchos, local de conservação de insulina, consultas extras e outros), $11,1 \%$ dias de doença, $8,1 \%$ hiperglicemia, $7,2 \%$ hipoglicemia, $12,2 \%$ ajuste de doses de insulina e $10,8 \%$ para obtenção de resultado de exames (Figura 2).

\section{Exames laboratoriais}

Os exames laboratoriais são coletados no prédio do ICD e processados no laboratório central do GHC. A hemoglobina glicada (Alc) é dosada inicialmente e a cada três meses, com valor de referência do serviço de $4,5 \%$ a $5,7 \%$, por imunoturbidimetria (Cobas Integra $700^{\circledR}$, Roche), padronizada pela NGSP (National Glycohemoglobin Standardization Program-USA). As metas da glicemia capilar e Alc adotadas no serviço, segundo as diferentes idades, estão descritas na tabela $\mathrm{l}$ e foram adaptadas de acordo com as recomendações da Associação Americana de Diabetes (ADA), da Associação Latino-Americana de Diabetes (ALAD), da International Diabetes Federation (IDF), da International Society for Pediatric and Adolescent Diabetes (ISPAD) e da Sociedade Brasileira de Diabetes (SBD) (1,11-14).

\section{Internações hospital-dia}

Internações hospitalares plenas

\begin{tabular}{|c|c|c|c|}
\hline 2004 & 2005 & 2006 & Out/2007 \\
\hline $\mathrm{n}=587$ & $\mathrm{n}=878$ & $\mathrm{n}=1.116$ & $\mathrm{n}=1.315$ \\
\hline 885 & 1.337 & 932 & 653 \\
\hline 44 & 36 & 34 & 35 \\
\hline
\end{tabular}

Percentual de internações hospitalares

$\left.\begin{array}{l|l|l}2004 & & 7,5 \% \\ 2005 & 4,1 \% & \\ 2006 & 3,0 \% \\ 2007 & 2,7 \%\end{array}\right\} \mathrm{P}<0,001$

Figura 1. Internações em hospital-dia e hospitalares plenas. 
Tabela 1. Metas da glicemia capilar e Alc adotadas no ICD de acordo com as faixas etárias.

\begin{tabular}{|c|c|c|c|c|c|}
\hline & $\begin{array}{l}\text { Pré-prandial } \\
\text { (mg/dl) }\end{array}$ & $\begin{array}{l}\text { Pós-prandial } \\
\text { (mg/dl) }\end{array}$ & $\begin{array}{l}\text { Ao deitar } \\
\text { (mg/dl) }\end{array}$ & $\begin{array}{l}\text { Madrugada } \\
\text { (mg/dl) }\end{array}$ & $\begin{array}{r}\text { Alc } \\
(\%)\end{array}$ \\
\hline 0 a 2 anos & $100-180$ & $<200$ & $120-200$ & $>100$ & $<8,5$ \\
\hline 3 a 6 anos & $90-150$ & $<200$ & $100-180$ & $>100$ & $<8,0$ \\
\hline 7 anos até puberdade & $70-150$ & $<180$ & $100-160$ & $>100$ & $<7,5$ \\
\hline Puberdade até adulto & $70-120$ & $<180$ & $80-150$ & $>80$ & $<7,0$ \\
\hline
\end{tabular}

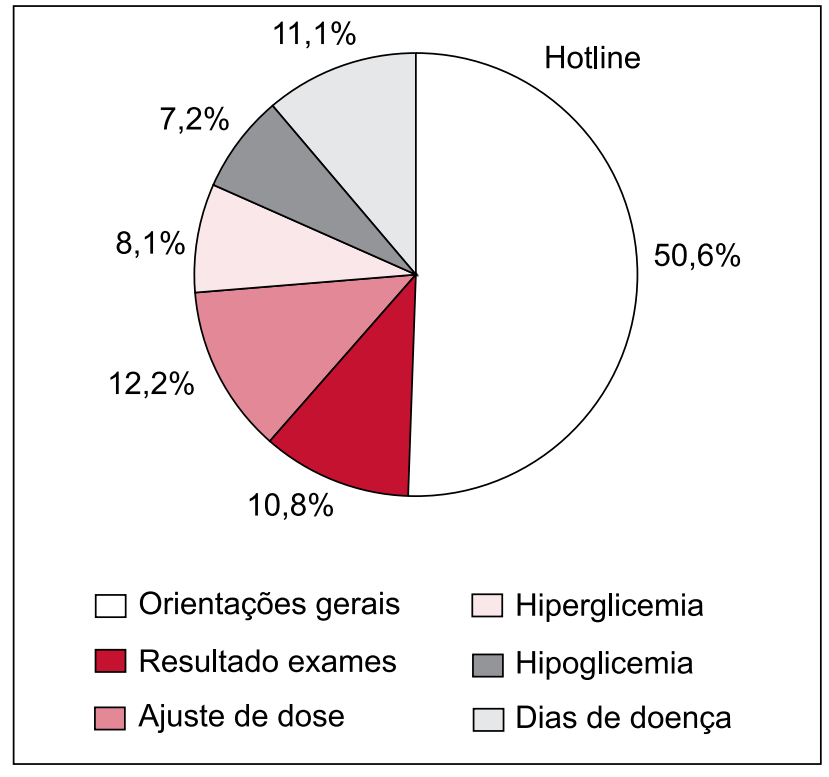

Figura 2. Motivos dos atendimentos telefônicos (Hotline).

$\mathrm{O}$ rastreamento de outras doenças auto-imunes, como a tireoidite de Hashimoto e a doença celíaca, é realizado rotineiramente, com dosagens anuais de TSH, anticorpos antiperoxidase $\mathrm{e} / \mathrm{ou}$ antimicrossomal e anticorpo antitransglutaminase, respectivamente. O TSH é determinado ao diagnóstico e após anualmente pela técnica de eletroquimioluminescência (ECLIA), com valor de referência de 0,27 a 4,2 $\mu \mathrm{UI} / \mathrm{ml}$, e os anticorpos antitiroperoxidase por método de microaglutinação passiva em partículas de gelatina (Microparticle Enzyme Immunoassay - MEIA). Em casos de TSH elevado, repete-se uma nova coleta do TSH associado ao $\mathrm{T}_{4}$ total (crianças) ou $\mathrm{T}_{4}$ livre (adolescentes, adultos) e, conforme resultado de hipotireodismo, inicia-se o tratamento com levotiroxina, com dosagens de TSH a cada seis meses, ou conforme necessidade de ajuste das doses de levotiroxina. Na presença apenas de anticor- pos positivos, aguarda-se a elevação do TSH ou o aparecimento de bócio para instituir o tratamento.

\section{Sistema de monitorização contínua de glicose subcutânea e bomba de infusão de insulina}

O serviço dispõe de sensores contínuos de glicose $\left(\right.$ CGMS $\left.^{\circledR}\right)$, os quais são instalados em pacientes que apresentam oscilações glicêmicas e/ou hipoglicemias severas recorrentes ou despercebidas, especialmente as noturnas. A identificação de padrões glicêmicos alterados permite ajustes na terapêutica com conseqüente melhora do controle metabólico. Até o dia 31 de outubro de 2007, foi instalado o CGMS $^{\circledR}$ em 69 pacientes. Nossa experiência mostrou que o CGMS $^{\circledR}$ é procedimento de fácil utilização na faixa etária pediátrica, com efeitos adversos de pequena intensidade, permitindo a identificação de padrões anormais de glicemia e ajustes no tratamento da maioria dos pacientes, com melhora no controle metabólico. Ao mesmo tempo, o prolongamento do teste (sete dias) permite uma análise preliminar dos dados, com observação dos potenciais benefícios das mudanças na terapêutica no tempo adicional de observação.

O ICD também disponibiliza seis bombas de infusão de insulina, que, com os insumos, são cedidas por um período de seis meses a pacientes previamente selecionados e, em casos de boa adaptação e melhora do controle metabólico, os pacientes são encaminhados à Secretaria da Saúde, visando a obtenção pelo Estado.

\section{Esquemas terapêuticos}

Em nosso serviço, o esquema terapêutico de insulinização dos indivíduos com DMl leva em consideração, inicialmente, a faixa etária ao diagnóstico do DM e os resultados das glicemias capilares pré-prandiais (desjejum, almoço e 
Tabela 2. Esquema de insulinização inicial recomendado no ICD em indivíduos que realizam automonitorização duas ou três vezes ao dia, de acordo com as faixas etárias.

Anos

0-2

3-6

7 anos até puberdade

Puberdade e adultos
Tipos e doses de insulina

$\mathrm{NPH}: 0,3 \mathrm{U} / \mathrm{kg} /$ dia $(2 \times$ dia: $2 / 3 \mathrm{AC}$ e $1 / 3 \mathrm{AD}$ ou glargina: $0,24 \mathrm{U} / \mathrm{kg} /$ dia $(1 \times$ dia, $\mathrm{AC}$ ) ou detemir: 0,3 U/kg/ dia (1 x dia, AC) associada à insulina de ação rápida ou ultra-rápida ( $A C, A A$ e AJ), de acordo ao resultado da glicemia capilar (> 250 mg/dl: $1 \mathrm{U}$ e a cada $100 \mathrm{mg} / \mathrm{dl}$ 1⁄2 U, máximo de 2 U/dose)

$\mathrm{NPH}: 0,3 \mathrm{U} / \mathrm{kg} /$ dia $(2 \times$ dia: $2 / 3 \mathrm{AC}$ e $1 / 3 \mathrm{AD}$ ) ou glargina: 0,24 U/kg/dia (1 × dia, AC) ou detemir: 0,3 U/kg/ dia (1 × dia, AC) associado à insulina ação rápida ou ultra-rápida: > 200 mg/dl: $1 \mathrm{U}$ e cada 50 mg/dl 1/2U, máximo $3 \mathrm{U} /$ dose (AC, $A A$ e AJ)

$\mathrm{NPH}: 0,3 \mathrm{U} / \mathrm{kg} / \mathrm{dia}(2 \times$ dia: $2 / 3$ AC e $1 / 3$ AD ou $3 \times$ dia: $1 / 2$ AC, $1 / 4$ AA e 1/4 AD) ou glargina: 0,24 U/kg/dia (1 x dia, $A C$ ) ou detemir: $0,3 \mathrm{U} / \mathrm{kg} /$ dia $(1 \times$ dia, $A C)$ associada à insulina de ação rápida ou ultra-rápida: > 150 mg/dl: 1 U e a cada 50 mg/dl 1/2 U, máximo 5 U/dose (AC, AA e AJ)

$\mathrm{NPH}: 0,5 \mathrm{U} / \mathrm{kg} /$ dia $(3 \times$ dia: $1 / 2 \mathrm{AC}, 1 / 4 \mathrm{AA}$ e 1/4 AD) ou glargina: 0,4 U/kg/dia $(1 \times$ dia, $\mathrm{AC}$ ) ou detemir: 0,5 U/kg/ dia (2 × dia: 2/3 AC e 1/3 AD) associada à insulina de ação rápida ou ultra-rápida: > 100 mg/dl: 1 U e a cada $50 \mathrm{mg} / \mathrm{dl} 1 \mathrm{U}$, máximo $8 \mathrm{U} /$ dose (AC, AA e AJ).

AC: antes do café, AA: antes do almoço, AJ: antes do jantar, AD: antes de deitar, U: unidades.

Tabela 3. Esquema de insulinização inicial recomendado no ICD, em indivíduos que não realizam automonitorização, de acordo com as faixas etárias.

\begin{tabular}{|c|c|}
\hline Anos & Tipos e doses de insulina \\
\hline $0-2$ & $\begin{array}{l}\text { NPH: } 0,3 \mathrm{U} / \mathrm{kg} / \text { dia }(2 \times \text { dia: } 2 / 3 \mathrm{AC} \text { e } 1 / 3 \mathrm{AD} \text { ou glargina: } 0,24 \mathrm{U} / \mathrm{kg} / \text { dia }(1 \times \text { dia, } \mathrm{AC} \text { ) ou detemir: } 0,3 \mathrm{U} / \mathrm{kg} / \\
\text { dia }(1 \times \text { dia, } \mathrm{AC}) \text { sem a associação da insulina de ação rápida ou ultra-rápida }\end{array}$ \\
\hline $3-6$ & $\begin{array}{l}\mathrm{NPH}: 0,5 \mathrm{U} / \mathrm{kg} / \mathrm{dia}(2 \times \text { dia: } 2 / 3 \mathrm{AC} \text { e } 1 / 3 \mathrm{AD}) \text { ou glargina: } 0,4 \mathrm{U} / \mathrm{kg} / \text { dia }(1 \times \text { dia, } \mathrm{AC}) \text { ou detemir: } 0,5 \mathrm{U} / \mathrm{kg} / \mathrm{dia} \\
(1 \times \text { dia, } \mathrm{AC}) \text { sem a associação da insulina de ação rápida ou ultra-rápida }\end{array}$ \\
\hline $\begin{array}{l}7 \text { anos até } \\
\text { puberdade }\end{array}$ & $\begin{array}{l}\mathrm{NPH}: 0,3 \mathrm{U} / \mathrm{kg} / \text { dia }(2 \times \text { dia: } 2 / 3 \mathrm{AC} \text { e } 1 / 3 \mathrm{AD} \text { ou } 3 \times \text { dia: } 1 / 2 \mathrm{AC}, 1 / 4 \mathrm{AA} \text { e } 1 / 4 \mathrm{AD}) \text { ou glargina: } 0,24 \mathrm{U} / \mathrm{kg} / \mathrm{dia}(1 \times \\
\text { dia, } \mathrm{AC} \text { ) ou detemir: } 0,3 \mathrm{U} / \mathrm{kg} / \mathrm{dia}(1 \times \text { dia, } \mathrm{AC}) \text { associada à insulina de ação rápida ou ultra-rápida: } 0,2 \mathrm{U} / \\
\mathrm{kg} / \text { dia ( } 3 \times \text { dia, } \mathrm{AC}, \mathrm{AA} \text { e } \mathrm{AJ})\end{array}$ \\
\hline $\begin{array}{l}\text { Puberdade e } \\
\text { Adultos }\end{array}$ & $\begin{array}{l}\mathrm{NPH}: 0,5 \mathrm{U} / \mathrm{kg} / \mathrm{dia}(3 \times \text { dia: } 1 / 2 \mathrm{AC}, 1 / 4 \mathrm{AA} \text { e } 1 / 4 \mathrm{AD}) \text { ou glargina: } 0,4 \mathrm{U} / \mathrm{kg} / \mathrm{dia}(1 \times \text { dia, } \mathrm{AC}) \text { ou detemir: } 0,5 \mathrm{U} / \mathrm{kg} / \\
\text { dia }(2 \times \text { dia: } 2 / 3 \mathrm{AC} \text { e } 1 / 3 \mathrm{AD}) \text { associada à insulina de ação rápida ou ultra-rápida: } 0,2 \mathrm{U} / \mathrm{kg} / \text { dia }(3 \times \text { dia, } \\
\mathrm{AC}, \mathrm{AA} \text { e AJ) }\end{array}$ \\
\hline
\end{tabular}

AC: antes do café, AA: antes do almoço, AJ: antes do jantar, AD: antes de deitar, U: unidades.

jantar), implementando-se desde o início o tratamento intensivo. Posteriormente, passado o período de lua-de-mel, ou na presença de hiperglicemias pós-prandiais, faz-se necessário o ajuste individualizado da titulação da insulina, de acordo com a idade, a insulina basal/bólus, a contagem de carboidratos, a relação insulina/carboidrato, o peso corporal e a atividade física.

As doses iniciais recomendadas em indivíduos que realizam automonitorização duas ou três vezes ao dia, conforme a faixa etária, estão descritas na tabela 2. Em casos de falta de disponibilidade de automonitorização, descritas na tabela 3 .

\section{Orientações em hipoglicemia}

A conduta diante dos episódios de hipoglicemias é de acordo com o nível da glicemia capilar, e os ajustes das doses de insulina são realizados posteriormente consoante a causa da hipoglicemia (associada a prática de atividade física, omissão ou atraso das refeições, excesso de insulina, erro de aspiração, consumo excessivo de bebidas alcoólicas).

Recomenda-se:

- Se a glicemia capilar estiver entre 50 e $70 \mathrm{mg} / \mathrm{dl}$ : antecipar a refeição (almoço ou jantar) ou oferecer 
um lanche (exemplo: leite e sanduíche com queijo e margarina).

- Se a glicemia capilar estiver $<50 \mathrm{mg} / \mathrm{dl}$ :

- Consciente (acordado): oferecer um líquido açucarado com aproximadamente 15 gramas de carboidratos (uma colher de sopa rasa de açúcar diluída em meio copo d'água, ou meio a um copo de refrigerante normal ou de suco de frutas, ou uma colher de sopa de mel ou um sachê de glicose). Repetir teste em aproximadamente $15 \mathrm{minu}-$ tos. Se glicemia $>50 \mathrm{mg} / \mathrm{dl}$ ou com melhora dos sintomas, antecipar a próxima refeição. Se glicemia $\leq 50 \mathrm{mg} / \mathrm{dl}$ ou não havendo melhora dos sintomas, repetir o líquido açucarado.

- Inconsciente (não responde a estímulos, muito sonolento): colocar mel ou açúcar (uma a duas colheres de sopa) na região interna da bochecha e da gengiva do paciente ou aplicar meia a uma ampola de glucagon $\left(\right.$ Glucagen $^{\circledR}$ ) via intramuscular. Após a melhora dos sintomas, liberar a refeição. Em casos de não-melhora ou presença de crise convulsiva, procurar atendimento médico de urgência ou contatar o ICD.

\section{PROGRAMA DE EDUCAÇÃO EM DIABETES E OFICINA DE NUTRIÇÃO}

No manejo interdisciplinar também está incluída a participação dos pacientes, familiares e/ou responsáveis, além de profissionais da área da saúde no Programa de Educação em Diabetes (PED), com aulas teórico-práticas diárias, de 45 minutos de duração, sobre noções gerais de diabetes, procedimentos diante de hipoglicemias, dias de doença e cetoacidose, aplicação e rodízio planejado de insulina, automonitorização, terapia nutricional e oficinas de nutrição, com orientações sobre preparo e qualidade dos diferentes alimentos, contagem de carboidrato, orientações gerais na escola e durante a prática de atividade física, novos avanços terapêuticos, mitos e crendices, entendendo as emoções e a prática de atividade física, incentivando o paciente e sua família ao melhor manejo e entendimento de sua doença. $\mathrm{O}$ cronograma das aulas é fixado nos painéis do ICD (Figura 3), com antecedência de um mês, para que os pacientes, familiares e interessados possam obter informações sobre o calendário das aulas, o qual também pode ser visualizado no site do ICD, conforme Figura 2.
O número total de participante no PED, a partir de novembro de 2005 , foi de 2.577 participantes (926 pacientes, 1.202 familiares e 449 outros profissionais da saúde), com uma freqüência de 6.906 participações (3.071 de pacientes, 3.093 de familiares e 742 de outros profissionais).

A oficina de nutrição é uma ferramenta didática utilizada no ICD para que os pacientes ou seus responsáveis adquiram maior conhecimento teórico-prático sobre alimentação saudável e autocuidado. São realizadas duas oficinas por mês, sempre às quartas-feiras à tarde (das 14 às 16 horas). A primeira aula mensal consiste em ensinar sobre alimentação correta, conforme a faixa etária, e adesão a novos hábitos saudáveis. Nessa aula, apresenta-se a pirâmide alimentar, orienta-se sobre a alimentação balanceada, valor nutricional dos alimentos, interpretação dos rótulos e a quantificação de alimentos industrializados e realizam-se preparações diet, light e pratos salgados. A segunda aula mensal é direcionada para o método de contagem de carboidratos, de maneira teórica e prática. Orientam-se para a contagem por gramas de carboidratos, a quantificação por meio da pesagem em gramas dos alimentos, o uso das tabelas de valores nutricionais dos alimentos (quantidade e qualidade) e o cálculo dos carboidratos ingeridos diariamente.

\section{Brinquedoteca}

A brinquedoteca do ICD oportuniza atividades lúdicoeducacionais às crianças, aos adolescentes e a seus responsáveis, durante o atendimento da equipe interdisciplinar, com o objetivo de auxiliar no atendimento clínico do diabetes e melhorar o controle e a aceitação da doença. Profissionais em formação, do curso de Pedagogia, realizam o atendimento lúdico-pedagógico, utilizando referenciais teórico-práticos associados à dinâmica de ensino e aos processos de aprendizagem. São oferecidas atividades livres, oficinas lúdico-pedagógicas (desenho, pintura, criação etc.) em atividades estruturadas, vinculadas a datas comemorativas (Dia da Criança, Natal, Dia dos Pais, Dia das Mães, Carnaval, Páscoa etc.). Esse espaço possui armários, brinquedos (carrinhos, bonecas, panelinhas etc.), diferentes tipos de jogos para as diversas idades, espaço para leitura, teatro, desenho, pintura, faz-de-conta. Também constituiu-se uma brinquedoteca itinerante, no intuito de dirigir-se aos locais em que as crianças e os jovens permanecem algum tempo aguardando os atendimentos, como a sala de espera do ambulatório ou o hospital-dia. 


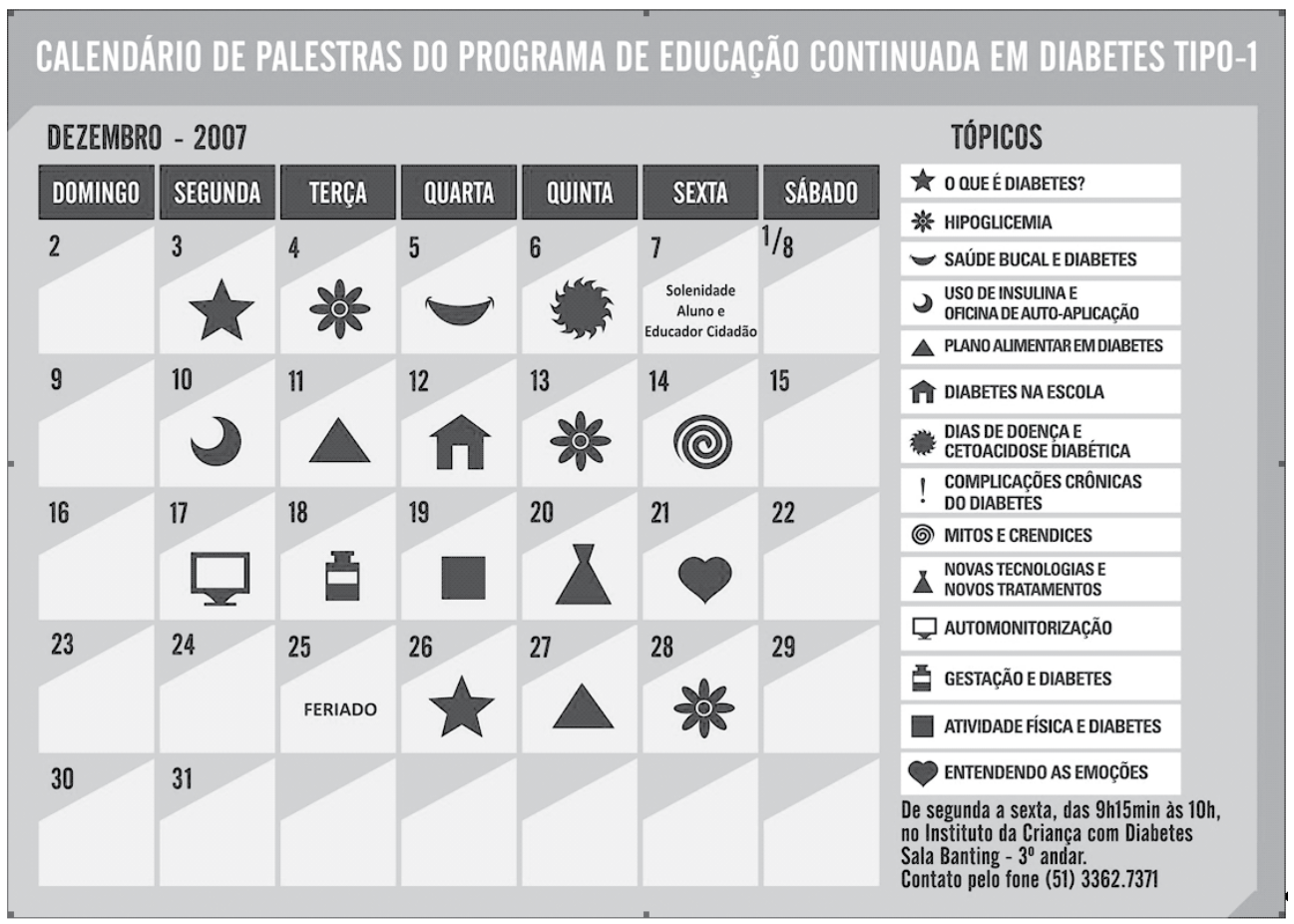

Figura 3. Calendário de palestras do Programa de Educação em Diabetes.

\section{Programas aluno-cidadão e professor-cidadão}

Os programas aluno-cidadão e professor-cidadão foram desenvolvidos com o objetivo de sensibilizar, fornecer informações sobre o diabetes e seu tratamento e capacitar multiplicadores de conhecimento, sendo dirigidos a estudantes e professores do ensino fundamental e médio da grande Porto Alegre. Às quartas-feiras, durante os turnos da manhã e da tarde, de quatro a seis estudantes ou professores de escolas previamente inscritas, acompanham e recebem orientações supervisionadas sobre as diversas atividades do ICD. Acompanhados pela equipe técnica interdisciplinar, conhecem o funcionamento do ambulatório, hospital-dia, biblioteca, oficina de nutrição e de insulina e brinquedoteca.

A cada trimestre é realizada uma solenidade de entrega oficial do título aluno-cidadão e professor-cidadão, com participação dos familiares, diretoria e equipe do ICD, diretoria das escolas, educadores, colegas e personalidades. O ICD entrega às escolas o troféu "Escola Amiga do Instituto da Criança com Diabetes". Até o momento, 45 escolas participaram dos programas, com 189 alunos e 61 professores participantes.

\section{Programa de estágio observacional}

O programa de estágio observacional é uma parceria entre o ICD e a indústria farmacêutica (Sanofi Aventis), propiciando a médicos endocrinologistas e outros profissionais de diferentes localidades do Brasil, durante um período de cinco dias, o acompanhamento de toda a dinâmica do ICD, capacitando profissionais na estruturação de um serviço multi e interdisciplinar em diabetes, visando a ampliar o conhecimento e o tratamento intensivo do DMl nas diversas regiões do país.

\section{Estágios curriculares, observacionais e trabalhos de conclusão}

O ICD propicia estágios curriculares a alunos (medicina, enfermagem, nutrição, psicologia, odontologia, educação física, pedagogia, serviço social) de instituições conveniadas com o Grupo Hospitalar Conceição e estágios observacionais a instituições não conveniadas. Além disso, o ICD permite a execução de trabalhos de conclusão de cursos (graduação e pós-graduação) com supervisão e orientações das diferentes áreas. 


\section{Dia de educação e lazer}

No final de cada ano, o ICD promove o dia de educação e lazer para pacientes interessados, sem a presença de familiares, propiciando a integração com a equipe interdisciplinar, em um local adequado para a realização de brincadeiras ao ar livre, atividades esportivas, oficinas de dança e equitação. Essas atividades são entremeadas com aspectos lúdico-educativos do dia-a-dia do diabetes, em parceria com outras instituições.

\section{DISCUSSÃO E CONCLUSÃO}

O modelo adotado pelo ICD, de uma organização do terceiro setor, em parceria com o serviço público (no caso, a União), permite obter as vantagens de um serviço público gratuito, aliadas às vantagens de captação de recursos do terceiro setor. Com isso, conseguimos atender um número expressivo de pacientes, todos em tratamento intensivo, aliando-se um programa educativo diário e contínuo, conforme o preconizado pela Society for Pediatric and Adolescent Diabetes (ISPAD) (12) à assistência interdisciplinar. A captação de recursos por parte do ICD permite alcançar insumos básicos, como tiras reagentes, até insulinas e tecnologias de última geração, aos pacientes mais carentes, pelo menos enquanto os mecanismos de obtenção desses materiais por meio do serviço público sejam providenciados pelos cuidadores destes.

Dos três objetivos principais do ICD, o primeiro, qual seja, o de diminuir a freqüência de hospitalizações por situações agudas, já está sendo alcançado, provavelmente fruto da atenção dispensada aos pacientes, como rápido acesso presencial ou telefônico, aliado ao processo educativo constante. O segundo objetivo, que é o de diminuir a freqüência de complicações crônicas, só poderá ser mensurado a médio e longo prazos. $\mathrm{O}$ terceiro objetivo, que é o de capacitar recursos humanos, está sendo cumprido por meio dos diversos programas que visam a capacitar não apenas médicos e pessoal técnico, mas também professores e alunos de escolas públicas e privadas, visando a ampla disseminação de conceitos sobre diabetes e, com isso, talvez até agilizando o diagnóstico de DMl, o que pode evitar que este seja feito apenas quando o quadro já evoluiu para cetoacidose (15).

Embora a literatura sobre o assunto específico deste artigo seja extremamente pobre, há alguns guidelines e referências pontuais que podem servir de orientação (11-13,16). Entretanto, sabendo-se das dificuldades de organização de um sistema de saúde eficaz no país, acreditamos que o modelo do ICD, que captura o essencial desses parâmetros, respeitando-se as diferentes particularidades regionais, possa ser mimetizado em diferentes capitais e cidades de médio porte, criando-se centros de tratamento intensivo para DM na infância e na adolescência, com educação constante e acesso fácil (telefônico, Internet ou presencial), visando principalmente a diminuição das taxas de internação, conforme dados apresentados no presente artigo.

Quanto ao modelo educativo seguido no ICD, embora possa haver alguma divergência eventual sobre retirada ou acréscimo de algum tema, parece não haver dúvidas da necessidade de que ele seja freqüente e constante (12). Para termos certeza de sua eficácia, o ideal seria compará-lo com outros programas de educação em diabetes. $\mathrm{O}$ atendimento no mesmo local por uma equipe interdisciplinar, com ambulatório e hospital-dia, permite que os pais/cuidadores não perambulem por filas e longas esperas, o que diminui a evasão. De qualquer modo, a assistente social faz um trabalho de busca ativa a todo o paciente que não comparece ao serviço há mais de seis meses. Todos sabemos que os pais de um paciente com DMl estão, em geral, em idade produtiva, e as dificuldades no acesso às consultas fazem que eles, muitas vezes, só procurem atendimento para seus filhos em situações agudas.

O ICD atende aproximadamente $40 \%$ da incidência estimada dos casos de DMl no Rio Grande do Sul, estando com 1.315 pacientes em atendimento atualmente. Esses números aumentam nossa responsabilidade e fazem que nos esforcemos cada vez mais para viabilizar o acesso irrestrito às novas tecnologias, enquanto elas não são absorvidas pelo setor público.

Nossos dados sugerem a importância do atendimento por uma equipe interdisciplinar em regime ambulatorial e de hospital-dia na atenção ao paciente com diabetes na infância e na adolescência, integrando a assistência a técnicas educativas diárias em uma estrutura pública, de fácil acesso, com suporte da comunidade, por meio de uma organização social não-governamental.

\section{REFERÊNCIAS}

1. IDF: Incidence of diabetes. Diabetes Atlas, 2006. Disponível em: http://www.eatlas.idf.org/incidence/

2. Diabetes control and complications trial research group. Effect of intensive diabetes treatment on the development and progression of long-term complications in adolescents with insu- 
lin-dependent diabetes mellitus: Diabetes Control and Complications Trial. J Pediatr. 1994;125:177-88.

3. Mensing C, Boucher J, Cypress M, Weinger K, Mulcahy K, Barta $\mathrm{P}$, et al. National standards for diabetes self-management education. Diabetes Care. 2005;28 Suppl 1:S72-79.

4. Clement S. Diabetes self-management education. Diabetes Care. 1995;18:1204-14.

5. Lorenz RA, Bubb J, Davis D, Jacobson A, Jannasch K, Kramer J, et al. Changing behavior. Practical lessons from the diabetes control and complications trial. Diabetes Care. 1996;19:648-52.

6. Murphy HR, Rayman G, Skinner TC. Psycho-educational interventions for children and young people with type 1 diabetes. Diabet Med. 2006;23:935-43.

7. Hampson SE, Skinner TC, Hart J, Storey L, Gage H, Foxcroft D, et al. Effects of educational and psychosocial interventions for adolescents with diabetes mellitus: a systematic review. Health Technol Assess. 2001;5:1-79.

8. Winkley K, Ismail K, Landau S, Eisler I. Psychological interventions to improve glycaemic control in patients with type 1 diabetes: systematic review and meta-analysis of randomised controlled trials. Britsh Med J. 2006;333:65.

9. Jacobson AM, Hauser ST, Willett J, Wolfsdorf JI, Herman L. Consequences of irregular versus continuous medical followup in children and adolescents with insulin-dependent diabetes mellitus. J Pediatr. 1997;131:727-33.

10. Kaufman FR, Halvorson M, Carpenter S. Association between diabetes control and visits to a multidisciplinary pediatric diabetes clinic. Pediatrics. 1999;103:948-51.
11. Hanas R, Donaghue K, Klingensmith G, Swift PG. ISPAD clinical practice consensus guidelines 2006-2007. Pediatr Diabetes. 2006;7:341-2.

12. Swift PG. Diabetes education. ISPAD clinical practice consensus guidelines 2006-2007. Pediatr Diabetes. 2007;8:103-9.

13. Standards of medical care in diabetes-2006. Diabetes Care. 2006; 29 Suppl 1:S4-42.

14. Sociedade Brasileira de Diabetes: posicionamentos oficiais da Sociedade Brasileira de Diabetes. Indicações de análogos de insulina de ação rápida e prolongada e de insulina inalável no tratamento do diabetes tipo 1 e tipo 2. Rev Bras Med. 2006; 2:7-11.

15. Vanelli M, Chiari G, Ghizzoni L, Costi G, Giacalone T, Chiarelli F. Effectiveness of a prevention program for diabetic ketoacidosis in children. An 8-year study in schools and private practices. Diabetes Care. 1999;22:7-9.

16. La Loggia A. Organization of paediatric diabetes units in Italy. Acta Biomed. 2005;76 Suppl 3:70-4.

\section{Endereço para correspondência:}

Balduino Tschiedel

ICD

Rua Álvares Cabral 529, Cristo Redentor

91350-250, Porto Alegre, RS

E-mail: tbalduino@ghc.com.br / http://www.icdrs.org.br 